\title{
Aprendizaje práctico de la bioética en el pregrado: objetivos, herramientas docentes y metodología
}

\author{
Azucena Couceiro
}

\section{Introducción}

El aprendizaje práctico de la bioética es un tema recurrente en la literatura. Pese a ello, resulta difícil encontrar un artículo que describa con precisión cómo hacerlo y, sobre todo, qué instrumentos utilizar para conseguir las habilidades y destrezas que se quieren desarrollar en el alumno.

La mayoría de los artículos inciden sobre aspectos metodológicos como el tamaño de los grupos, el uso de casos clínicos para ubicar los dilemas éticos de la práctica real, o la discusión posterior acerca de los problemas éticos detectados [1]. De esta manera se intenta aplicar el aprendizaje basado en problemas al ámbito de la ética. Si bien esta es una aproximación metodológica correcta, el problema reside en que, en general, no se ofrecen más herramientas prácticas que la discusión de casos o la utilización de películas en las que aparecen conflictos éticos, que se analizan y discuten en pequeños grupos.

Este uso docente, así planteado, mantiene algunos de los errores conceptuales típicos de la enseñanza de la ética, a saber:

- Basta con que los alumnos debatan y expresen libremente sus opiniones sobre los conflictos de valores que acaecen en el caso que se les presenta.

- El objetivo es un intercambio de opiniones y argumentos, puesto que todas las opiniones deben ser escuchadas en un marco docente que asume la 'neutralidad' axiológica.

- En todo caso, es suficiente con que los alumnos identifiquen problemas y los ubiquen bajo el paradigma de los cuatros principios de la bioética [2].

En este artículo se presenta un diseño metodológico práctico, adecuado para la adquisición de habilidades concretas, que evita las deficiencias señaladas previamente. En la primera parte se proponen herramientas y materiales de trabajo en relación con las competencias a desarrollar en la docencia de la bioética en el pregrado [3]. En la segunda se explicita cómo se tiene que proceder para que en el diálogo posterior, en grupos reducidos, se llegue a una auténtica deliberación, que es la habilidad práctica de mayor importancia en el ámbito de la ética.

Se deja de lado el uso del cine como recurso para el aprendizaje de la bioética por entender que ha sido el más y mejor desarrollado en la bibliografía $[4,5]$. Además, en el pregrado, debería ser una herramienta más que sirviera de apoyo a un proceso de aprendizaje diseñado en torno a competencias específicas, pero que no se redujera sólo a este instrumento docente.

\section{Bioética y relación clínica}

Partimos de la base de que el diseño curricular de la enseñanza de la bioética es similar al del resto de las asignaturas, que se estructuran alrededor de dos ciclos: básico y clínico. Este es el modelo que desde principios del siglo pasado ha marcado el aprendizaje de casi todos los médicos del mundo [6,7].

En la etapa preclínica o básica se debe enseñar bioética básica, cuyos contenidos giran alrededor de la ética y la relación clínica: cómo surge, qué significa que la bioética es ética cívica, cuáles son los principios de la bioética y qué significan, cuáles son los modelos de relación clínica y cuáles de ellos permiten el respeto de los pacientes y de sus derechos, qué es el proceso de consentimiento y cuáles sus elementos, cómo se razona metodológicamente en ética, etc. [8].

En la delimitación de las actividades prácticas, y para centrar su diseño, hay que relacionar los contenidos teóricos (saber) con las destrezas (saber hacer) que deben adquirir los alumnos. En esta asignatura, 'Bioética y relación clínica', se pueden señalar las siguientes destrezas y habilidades:

- Identificar el papel de cada uno de los actores de la relación clínica en la sociedad actual.
Área de Bioética. Departamento de Especialidades Médico Quirúrgicas. Facultad de Medicina. Universidad del País Vasco (UPV/ EHU). Leioa, Vizcaya, España.

Correspondencia:

Dra. Azucena Couceiro Vidal. Área de Bioética. Departamento de Especialidades Médico Quirúrgicas. Facultad de Medicina. Universidad del País Vasco (UPV/EHU). Barrio Barriera, $\mathrm{s} / \mathrm{n}$. E-48940 Leioa (Vizcaya).

E-mail:

acouceiro@arrakis.es

Conflicto de intereses: No declarado.

Conflict of interests: None declared.

(c) 2012 Educación Médica 
Tabla I. Bioética básica: práctica 1 .

Objetivos

1. Analizar la sociedad española desde un punto de vista axiológico.

2. Analizar si es -o no- una sociedad democrática plural, respetuosa con la libertad, respetuosa de las diversas creencias religiosas, solidaria en la distribución de la riqueza y del acceso a educación y sanidad, etc.

3. Comparar con lo que ocurre en otras sociedades contemporáneas con similar tradición histórica.

4. Analizar la relación clínica: cómo es y su relación con el tipo de sociedad en la que tiene lugar.

5. Identificar los problemas que plantea al profesional de la medicina la incorporación de estos nuevos elementos en la relación clínica.

\section{Material de apoyo}

Obtenido de periódicos y medios de comunicación, sobre temas axiológicos objeto de debate en la sociedad:

1. Díaz-Salazar R. ¿Es necesaria una asignatura de educación para la ciudadanía? El País, 11 de diciembre de 2005.

2. Otaola J. Dignidad de la fe, dignidad del ateísmo. El País, enero de 2009.

Indicaciones

Además del material de apoyo, los alumnos pueden utilizar cualquier otro texto o método (entrevistas a personas de diversas edades y profesiones, a médicos en ejercicio) que les permita generar argumentos y obtener datos para responder a la pregunta sobre cómo es, desde el punto de vista de la ética cívica, la sociedad española actual.

- Identificar el tipo de relación clínica en el que los profesionales se mueven habitualmente en función de la sociedad en la que viven, y cuál es el modelo de relación que permite respetar los derechos de los pacientes.

- Ser capaz de generar un proceso de consentimiento informado.

- Ser capaz de analizar críticamente los modelos (formularios) de consentimiento y saber cuáles constituyen una ayuda en el proceso comunicativo.

- Ser capaz de registrar adecuadamente el proceso de consentimiento en la historia clínica.

- Aprender cómo se evalúa la capacidad de los pacientes para tomar decisiones y cómo se registra dicha evaluación en la historia.

Su consecución se puede articular en torno a tres prácticas, que inciden en los conceptos y áreas de trabajo fundamentales en esta asignatura: relación clínica y ética civil, el proceso de consentimiento informado, y el rechazo del tratamiento por parte del paciente y el problema de la capacidad. Cada una de estas prácticas se debe acompañar de un guión para los alumnos que señala los objetivos, facilita el material bibliográfico sobre el que tienen que elaborar su trabajo práctico, y proporciona las indicaciones específicas para su manejo y el cumplimiento de los objetivos señalados.

Para el diseño de herramientas de aprendizaje no podemos olvidar que el alumno todavía no tiene experiencia clínica, de forma que cuando se utilice el recurso del caso clínico éste no deberá contener muchos datos, ni excesivamente complejos. En todo caso, será muy sencillo y previamente explicado en todos sus aspectos -diagnósticos, pronósticos y terapéuticos- por la persona que tutoriza el aprendizaje.

\section{1. ํ. práctica: relación clínica y ética civil}

Si hay una noción fundamental en el aprendizaje de la bioética es la noción de ética civil. La bioética es un tipo de ética aplicada que presupone la introducción en la medicina de los valores democráticos que fundamentan la vida civil de las sociedades occidentales [9].

Irrumpe en la relación clínica de la mano de la sociedad que, por una parte, exige el respeto a la autonomía del ciudadano, y al mismo tiempo conceptúa el acceso a la asistencia sanitaria como una cuestión de justicia distributiva, que la sociedad debe procurar a todos los individuos [10].

El alumno debe reflexionar y utilizar los contenidos previamente explicados en las clases teóricas para ubicar el tipo de sociedad civil en la que vive y llegar a relacionar ésta con el modelo de relación clínica que manejan con más frecuencia los profesionales (Tabla I). El objetivo es identificar que hay modelos que permiten el respeto de los derechos de los pacientes y el desarrollo del proceso comunicativo del consentimiento informado, y otros, como el modelo paternalista, que anulan esa posibilidad.

Para adquirir esta destreza y analizar la sociedad civil nada mejor que utilizar el material que proporciona la propia sociedad. A modo de ejemplo, los periódicos son el reflejo directo de la vida civil de un país, de los temas que le preocupan, y de la forma en que se analizan (argumentos emotivos, argumentos racionales, argumentos de poder, etc.) los hechos que acaecen. En principio es recomendable evitar noticias que se refieran directamente al mundo sanitario, o al menos no utilizar exclusivamente materiales en relación con este ámbito, ya que el objetivo es trabajar la conformación axiológica de la sociedad civil para comprobar, en un paso posterior, que la relación clínica no es sino un mi- 
crocosmos en el que se refleja la estructura de valores de la sociedad en general.

\section{2. a práctica: proceso de consentimiento informado}

El modelo deliberativo de relación clínica exige poner en práctica procesos de consentimiento informado [11]. Para adquirir dicha habilidad es necesario conocer los elementos del proceso -información, capacidad y voluntariedad-, utilizar capacidades comunicativas y, sobre todo, no confundir los formularios escritos con los procesos clínicos de toma de decisiones consensuadas. Este es el objetivo prioritario de la práctica (Tabla II).

El análisis de diversos formularios es un excelente instrumento de aprendizaje práctico. El tutor debe hacer una selección para presentar al alumno formularios bien diseñados, que contienen todos los elementos informativos, y que cumplen tanto los criterios de contenido como los de legibilidad. El alumno debe compararlos con formularios mal realizados, genéricos, no comprensibles o que contienen elementos que no corresponden, como que 'la firma de los mismos exime de responsabilidad al profesional, o afirmaciones similares.

La práctica puede complementarse con artículos sobre el consentimiento. Se recomienda no utilizar artículos genéricos, que en todo caso ya se habrán trabajado en la parte teórica correspondiente a este tema, sino aquellos publicados en revistas clínicas de prestigio, con un buen diseño metodológico, que ofrezcan investigaciones empíricas. Sus resultados ilustran al alumno acerca de los problemas reales que acaecen en la praxis clínica y le permiten reflexionar sobre las dificultades que este proceso entraña para los clínicos y para los pacientes.

\section{3. a práctica: rechazo del tratamiento y evaluación de la capacidad}

Una de las situaciones más complejas en las que se puede encontrar el profesional de la salud es la que se produce cuando un paciente rechaza el tratamiento que se le propone [12]. Esta era una actuación impensable, ética y jurídicamente, durante siglos, pero ahora se reconoce como uno de los derechos más importantes del paciente porque le otorga la capacidad decisoria final sobre su vida $[13,14]$.

El alumno tiene que conocer y comprender la razón de este derecho, tema objeto de las clases teóricas, pero su puesta en práctica conlleva ciertas habilidades profesionales que van más allá de la trasmisión de la información y la buena comunicación. Todos los actos de relevancia jurídica -y el rechazo
Tabla II. Bioética básica: práctica 2.

\section{Objetivos}

1. Identificar el proceso del consentimiento informado como una herramienta básica en la relación clínica actual.

2. Conocer y aplicar los elementos de ese proceso.

3. Identificar la información como elemento clave de relación con el paciente y la necesidad de poseer habilidades profesionales comunicativas (utilizar el texto de apoyo n.ㄹ 2).

4. Distinguir con claridad el proceso de comunicación del formulario escrito.

5. Saber realizar un buen formulario escrito de consentimiento.

6. Saber utilizar el formulario como instrumento de apoyo en el proceso comunicativo y no como mero trámite legal (utilizar el texto de apoyo n.우).

Materiales de apoyo

1. Ley $41 / 2002$, de 15 de noviembre, reguladora de la autonomía del paciente y de derechos y obligaciones en materia de información y documentación clínica. Boletín Oficial del Estado 2002; 274: 40126-32.

2. Simón $P$, et al. ¿Querría usted saber que tiene cáncer? Medifam 1994; 4: 127-36.

3. Hernando P. Influencia de un formulario escrito de consentimiento informado para biopsia hepática en la información y la ansiedad de los pacientes. Rev Clin Esp 1997; 197: 81.

4. Diversos formularios de consentimiento informado (para procedimientos diversos y de países distintos) que deben analizarse para identificar cuáles son correctos y de utilidad en el proceso comunicativo, y cuáles no.

Indicaciones

La práctica tiene dos partes, bien diferenciadas:

1. En la primera se debe trabajar el consentimiento informado como proceso, utilizando los conocimientos teóricos, y los documentos de apoyo 1 y 2 . Asimismo es importante que pregunten en su entorno, bien a familiares, bien a profesionales de la medicina, qué es lo que se hace en la realidad clínica. También que realicen una encuesta, tomando como base la que aparece en el

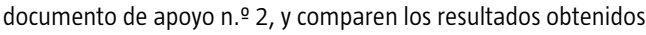
por los alumnos con los que aparecen en el citado artículo, intentando dar razón de las similitudes y diferencias.

2. En la segunda deben trabajar el formulario como instrumento de ayuda, para lo cual han de analizar y comparar los formularios de diversas instituciones para responder a las siguientes cuestiones:

- Si contienen toda la información necesaria, tanto médica como de identificación del centro, procedimiento, paciente médico, etc.

- Si la información es sencilla y clara, con términos comprensibles para los pacientes.

- Si existe la posibilidad de añadir información 'personalizada' del paciente.

- Analizar de manera específica el formulario sobre limitación del esfuerzo terapéutico: contenido, utilidad, sentido, claridad, etc., teniendo en cuenta los elementos teóricos ya estudiados. 
Tabla III. Bioética básica: práctica 3.

\section{Objetivos}

1. Identificar el derecho del paciente a rechazar tratamientos.

2. Conocer cuáles son los requisitos de capacidad necesarios para respetar sus elecciones, que deben ser evaluados por los médicos.

3. Determinar el papel de los familiares y allegados.

\section{Material de apoyo}

1. Ley $41 / 2002$, de 15 de noviembre, reguladora de la autonomía del paciente y de derechos y obligaciones en materia de información y documentación clínica. Boletín Oficial del Estado 2002; 274: 40126-32.

2. Etchels E, et al. Assessment of patient capacity to consent to treatment. J Gen Intern Med 1999; 14: 27-34. ${ }^{a}$

3. Appelbaum PS. Assessment of patient's competence to consent treatment. N Engl J Med 2007; 357: 1834-40.

4. Casos clínicos.

\section{Indicaciones}

Los alumnos deben comparar los casos facilitados en el material de apoyo, analizarlos y determinar si los pacientes son o no capaces de rechazar el tratamiento. Además, deben señalar posibles alternativas de tratamiento para los pacientes.

a Protocolo disponible en: http://www.utoronto.ca/jcb/disclaimers/ace.htm. Singer P. Guión de evaluación de la capacidad. Centro de Bioética de la Universidad de Toronto. Traducción española y modificación: Pablo Simón, Escuela Andaluza de Salud Pública. forma se consigue la correlación entre el aprendizaje de los hechos técnicos con la identificación de los problemas éticos más frecuentes en el ámbito del final de la vida.

El primer paso consiste en delimitar las habilidades (saber hacer) que deben desarrollar los alumnos:

- Identificar correctamente las situaciones o escenarios clínicos que aparecen en el proceso de muerte: la limitación del esfuerzo terapéutico, el rechazo de tratamiento, la sedación del enfermo en fase terminal o sedación paliativa, la suspensión de atención medica por fallecimiento tras el diagnóstico de muerte encefálica, y la eutanasia [15].

- Formular juicios clínicos argumentados sobre la futilidad en las intervenciones sanitarias y sobre la necesidad de limitarlas para evitar la obstinación diagnóstica o terapéutica.

- Aprender a realizar con los pacientes una planificación anticipada de sus decisiones y a reflejarla en la historia clínica.

- Identificar el marco clínico de los cuidados paliativos -síntomas prevalentes en las enfermedades avanzadas y en las enfermedades oncológicas terminales-y los problemas éticos asociados.

Para su consecución se pueden realizar tres prácticas que giran alrededor de tres ejes clínicos cuyos aspectos conceptuales deben haberse desarrollado previamente en las clases teóricas. Los ejes o escenarios clínicos son: el de las enfermedades agudas y enfermedades críticas, el de las enfermedades crónicas, y el abordaje paliativo de cualquier patología grave y avanzada que conlleve un proceso de muerte cercano o que ya lo haya iniciado.

\section{1. a práctica: enfermedades críticas; adecuación del esfuerzo terapéutico y comunicación con el paciente y los familiares}

En medicina, la aparición y uso de la tecnología va ligado a dos conceptos: el de enfermedad crítica y el que define y entiende la técnica como un fin en sí misma. Ambos son erróneos. Ni la tecnología afecta sólo a la enfermedad crítica -todo lo que se utiliza para el tratamiento de los enfermos, desde la diálisis renal hasta una simple sonda nasogástrica, constituyen posibilidades tecnológicas de intervención en el ser humano- , ni la técnica es un fin en sí misma, sino un medio cuyo uso debe valorarse en relación con cada caso clínico y su pronóstico.

Este cambio ha propiciado la aparición de conceptos como el de la limitación del esfuerzo terapéutico [16-18], hoy constitutivo de una buena práctica clínica, y que nada tienen que ver con la euta- 
Tabla IV. Casos clínicos para la evaluación de la capacidad de hecho.

\section{Caso 1: neoplasia de pulmón}

Paciente de 35 años, diagnosticado de un carcinoma pulmonar. Acude al consultorio por dolor costal de gran intensidad. La radiografía de tórax muestra fractura del sexto arco costal, producida por una metástasis del tumor primario.

El paciente proviene de Oncología, donde tras el diagnóstico se le ofreció tratamiento con quimioterapia para reducir el volumen de la neoplasia, sabiendo que por lo avanzado de la patología (al momento del diagnóstico presenta metástasis) y por el tipo histológico del carcinoma no se conseguirá la remisión del tumor, sino a lo máximo un incremento en la supervivencia de seis meses, y un control de los síntomas que aparecerán por el crecimiento del tumor y la compresión de estructuras adyacentes.

La Dra. Gómez establece con el paciente un diálogo en el que retoma la información y le pregunta por su decisión. El paciente se muestra claro y repite lo que ya dijo al oncólogo: que no quiere padecer los efectos secundarios de la quimioterapia, efectos que conoce a través de la experiencia de un familiar cercano que murió de cáncer un año antes, y que sabe que someterse al tratamiento no logrará la remisión del tumor, sino sólo una sobrevida estimada entre seis meses y un año.

El paciente se muestra cabizbajo. Es acompañado por su esposa y un hermano, que continuamente asienten y refuerzan la decisión del paciente, y añaden que van a consultar con un especialista en medicinas alternativas y con un acupuntor. La doctora le pide que reevalúe su decisión y que en todo caso vuelva por el consultorio cuando lo necesite.

Tres meses más tarde, el paciente acude de nuevo a la consulta con un gran cortejo sintomático: exoftalmos, ingurgitación venosa, dolores opresivos en el tórax y dificultades en la deglución. Se le ofrece un tratamiento de quimioterapia, que el paciente rechaza de nuevo, por lo que se establece comunicación con el equipo de cuidados paliativos con el objetivo de controlar los síntomas y acompañar la muerte del paciente, a ser posible en su domicilio.
La doctora se queda pensando que nunca antes había tenido un caso similar, y aunque intuye que no se debería forzar a nadie a seguir un tratamiento que no acepta, se siente inquieta porque no sabe cómo justificar su decisión, y tampoco si en este caso debía haber presionado al paciente para que aceptara la quimioterapia.

\section{Caso 2: fractura de cadera}

Paciente de 67 años, sin antecedentes de interés, que comienza en su domicilio con fiebre y dificultad respiratoria. Sufre una caída y una fractura consecuente, lo que le ocasiona gran dolor e impotencia funcional de la extremidad inferior izquierda. Es trasladada desde su domicilio al hospital comarcal más cercano.

Al ingreso, tras anamnesis, exploración y petición de pruebas complementarias, se realizan los siguientes juicios diagnósticos: neumonía extrahospitalaria y fractura pertrocantérea de la cadera izquierda.

Al ser informada de su situación clínica, así como de la necesidad de intervenirla quirúrgicamente, la paciente se niega a operarse y a recibir la heparina que, en caso de inmovilización prolongada, habría que pautarle para evitar un tromboembolismo pulmonar secundario.

Al preguntarle los motivos de su decisión argumenta que se cayó porque perdió el conocimiento debido a su proceso respiratorio, y que cuando éste revierta, desaparecerá también su problema de deambulación. Por esta razón acepta ser tratada con antibióticos y oxígeno, pero no la intervención quirúrgica.

El medico responsable habla con ella, le informa de nuevo y le muestra la radiografía para que objetive la fractura de cadera, pero la paciente repite todos los argumentos antes reseñados. Por esta razón, el médico tratante consulta al comité de ética para que analice el caso y le proporcione elementos para saber si debe respetar la decisión de la paciente. nasia. Es fundamental que los alumnos desarrollen la habilidad de enfocar la tecnología en el contexto del pronóstico del paciente y que aprendan a realizar juicios clínicos de limitación del esfuerzo terapéutico [19]. También lo es que aprendan a manejar correctamente el proceso comunicativo con pacientes y familiares en estas situaciones, y que puedan diseñar formularios sencillos que los ayuden en la transmisión de la información.

De nuevo se requiere un material de trabajo práctico, publicado en revistas clínicas de prestigio, y estudios empíricos que permitan que el alumno visualice la importancia de este tema en la praxis clínica y las dificultades de los profesionales para realizar estos juicios (Tabla V).

\section{2. práctica: enfermedades crónicas; planificación anticipada de las decisiones y directivas previas}

La planificación anticipada de las decisiones es una valiosa herramienta clínica en las enfermedades crónicas, avanzadas, terminales, etc. En realidad no es sino un proceso de consentimiento informado que se proyecta hacia el futuro, para poder tomar decisiones en representación del paciente cuando éste no sea capaz de decidir por sí mismo [20].

En esta práctica, y supuestos ya los conocimientos teóricos del tema, el alumno debe aprender a realizar un proceso de planificación y a aplicarlo a una situación clínica a través del caso que elija de entre el tipo de enfermedades mencionadas (Tabla VI). La tutorización del profesor lo ayudará a seleccionar el caso, a conocer su evolución y a identificar los episodios que ocurrirán en el futuro, y que deben comunicarse y dialogarse con los pacientes para recabar su posición respecto a la intervención clínica o tratamiento [21].

Además de este aprendizaje, otro objetivo de la práctica es disolver la confusión existente entre el formulario de instrucciones previas y el proceso de 
Tabla V. Bioética y final de la vida: práctica 1.

Objetivos

1. Identificar situaciones críticas en las que se requiere el uso de medios de soporte vital.

2. Analizar críticamente el manejo habitual de estos pacientes en las unidades de medicina intensiva.

3. Realizar una simulación de limitación del esfuerzo terapéutico (seleccionar un caso clínico, ubicarlo en uno de los paradigmas de actuación explicados para la imitación del esfuerzo terapéutico en medicina crítica, y llevar a cabo un análisis del mismo).

4. Conocer los resultados de las encuestas realizadas a los profesionales sobre la imitación del esfuerzo terapéutico.

5. Obtener datos empíricos encuestando a dos tipos de población: profesionales de salud y pacientes que hayan estado en la Unidad de Cuidados Intensivos (o sus familiares).

6. Elaborar un formulario de información para los familiares de pacientes ingresados en una Unidad de Cuidados Intensivos.

\section{Material de apoyo}

1. Fernández R, Baigorri F, Artigas A. Limitación del esfuerzo terapéutico en Cuidados Intensivos. ¿Ha cambiado en el siglo xxI? Med Intensiva 2005; 29: 338-41.

2. Gómez-Rubí J. Estrategia práctica de limitación del esfuerzo terapéutico en la Unidad de Cuidados Intensivos. In Gómez-Rubí J. Ética y medicina crítica. Madrid: Triacastela; 2002. p. 242-53.

3. Santana $L$, et al. Encuesta de satisfacción a los familiares de pacientes críticos. Med Intensiva 2007; 31: 57-61.

4. Pérez-Cárdenas MD, et al. Valoración del grado de satisfacción de los familiares de pacientes ingresados en una unidad de cuidados intensivos. Med Intensiva 2004; 28: 237-49.

5. Documento de 'Información a familiares y allegados de pacientes ingresados en la UCl'. Castellón: Hospital General de Castellón.

Indicaciones

Además de los textos específicos de soporte para esta práctica, los alumnos deben utilizar el material bibliográfico de la asignatura (parte teórica) correspondiente a este tema. La práctica tiene dos partes:

1. En la primera hay que aplicar el concepto de limitación del esfuerzo terapéutico al caso seleccionado, y ubicarlo en uno de los grupos de clasificación de limitación del esfuerzo terapéutico en medicina crítica.

2. En la segunda se debe trabajar el proceso de información a los familiares, identificando varios aspectos: de qué es necesario informar, cuándo hay que informar, a quién, y cómo utilizar instrumentos facilitadores para la comprensión de la información.

planificación anticipada de las decisiones. Al igual que ocurre con el consentimiento, el proceso de planificación debería hacerse siempre en este tipo de patologías, exista o no un documento de instrucciones previas [22].

\section{3. a práctica: enfermedades en fase avanzada y enfermedades terminales; abordaje paliativo}

Los cuidados paliativos nacieron en torno a la oncología y ahí es donde cualquier profesional sanitario los ubica como una parte más de la medicina, que entiende que el cuidado del proceso de la muerte también forma parte de las obligaciones de los profesionales [23]. Hoy el abordaje paliativo es mucho más amplio y se aplica también a las fases avanzadas de cualquier patología grave e irreversible.

Desde los inicios, esta especialidad ha convivido con numerosos problemas éticos, relacionados con la cercanía de la muerte y con su posible aceleración en el intento de paliar los síntomas del paciente. El uso de opiáceos para el control del dolor y sus efectos secundarios es uno de ellos. El otro, la sedación del enfermo terminal cuando hay síntomas refractarios que no responden al tratamiento [24,25].

En este nivel docente, el objetivo es que el alumno identifique el abordaje de los cuidados paliativos como una exigencia profesional, que conozca la obligación de tratar los síntomas de los pacientes, y que ubique los protocolos y guías de práctica clínica para el manejo del dolor y la sedación terminal (Tabla VII). Asimismo, que no confunda ninguna de estas actuaciones clínicas con la eutanasia.

Una vez más, los textos de apoyo deben ser muy sencillos, a la vez que clínicos. Las guías sobre cuidados a enfermos oncológicos y no oncológicos, editadas por las consejerías de salud, o los estudios empíricos publicados en revistas de la especialidad son una excelente herramienta práctica.

\section{Segunda fase: del mero intercambio de opiniones a la deliberación ética}

Cada una de las prácticas descritas se desarrolla en dos fases: la del trabajo personal del alumno a través del material que se le ha proporcionado, y la del diálogo posterior con el resto del grupo. Lo habitual en este tipo de diálogos es que se reúna el grupo y se escuchen todas las opiniones, mientras que el docente intenta ser lo más 'neutral' posible respecto a las cuestiones axiológicas planteadas por los alumnos. Es lo que se denomina 'pragmatismo educativo, un error bastante frecuente de la metodología docente en el área de los valores.

El origen de tal confusión se encuentra en nuestra sociedad, que ha convertido en sinónimos dos conceptos que no lo son: el de 'tolerancia' y el de 'neutralidad axiológica' De aquí la afirmación de que en una sociedad plural cualquier opinión es respeta- 
ble, y por ello hay que ser neutral respecto de todas ellas. El ámbito docente no es sino un microcosmos de esa sociedad, en el que aparece la misma idea.

Durante siglos, la enseñanza de los valores se ha basado en el puro 'dogmatismo' y así se ha mantenido en las sociedades jerárquicas. La evolución social hacia el pluralismo y la tolerancia ha llevado esta enseñanza justo hacia el otro extremo, hacia el 'pragmatismo educativo', que consiste en la mera resolución de problemas y afirma que sobre cuestión de valores hay que ser 'neutral', que todas las opiniones son válidas y que deben respetarse en aras de la misma tolerancia.

Pensemos en un paciente que rechaza un tratamiento prescrito por el clínico, tratamiento con importantes riesgos secundarios asociados pero, al mismo tiempo, con alta probabilidad de mantener la vida del paciente con una calidad aceptable. La educación dogmática inculcaría una respuesta al alumno que no puede discutirse porque corresponde a la valoración social de 'lo correcto'. La respuesta es que no es posible aceptar el rechazo de un tratamiento por parte del paciente, que quien rechaza un tratamiento sufre alguna alteración mental o afectiva, y que de cualquier forma el médico debe tratarlo de inmediato, aun en contra de su voluntad.

La educación en el pragmatismo, justo en el otro extremo, intentaría resolver el problema tras escuchar a todos, pero sin entrar a discutir ni posicionarse sobre qué se considera lo mejor desde el punto de vista axiológico o de los valores, quizás por el miedo a volver a caer en la posición dogmática que durante siglos ha caracterizado esta enseñanza. Se discute entre los alumnos, cada cual emite una opinión, y aunque sean encontradas y puedan llevar a paradojas, poco más se puede hacer en el ámbito de los valores. De hecho, si algo ha caracterizado a la bioética en sus inicios ha sido el pragmatismo liberal y una cierta neutralidad ideológica [26,27].

Hay una tercera alternativa que supera las deficiencias de los paradigmas previos, la educación en el modelo deliberativo. Deliberar significa analizar hechos, valores en conflicto, circunstancias y consecuencias previsibles, y así ponderar y tomar decisiones prudentes, que podamos sustentar con argumentos [28]. No se puede deliberar si no se conocen los hechos -situación clínica del paciente, pronóstico con y sin tratamiento, alternativas a ese tratamiento-, si no se identifican los valores en conflicto, si no se tienen elementos de análisis, que el alumno habrá adquirido en las clases teóricas. No se puede deliberar en serio si el alumno no sabe si en la sociedad en la que vive se reconoce al paciente ese derecho, si este reconocimiento tiene algún fun-
Tabla VI. Bioética y final de la vida: práctica 2.

Objetivos

1. Identificar aquellos escenarios clínicos en los que es conveniente planificar con el paciente decisiones futuras sobre el nivel de su tratamiento médico.

2. Conocer el contenido de la Ley 41/2002 acerca de las directivas previas, su uso en la práctica asistencial y sus límites.

3. Investigar las actitudes de pacientes y profesionales respecto a este tema.

4. Aprender cómo se lleva a cabo una planificación anticipada de las decisiones. Seleccionar un caso clínico y aplicar sobre él una planificación anticipada de decisiones, siguiendo el modelo que aparece en el artículo n. 05 del soporte bibliográfico.

Material de apoyo

1. Ley $41 / 2002$, de 15 de noviembre, básica reguladora de la autonomía del paciente y de derechos y obligaciones en materia de información y documentación clínica. Boletín Oficial del Estado 2002; 274: 40126-32.

2. Santos de Unamuno C. Documento de voluntades anticipadas: actitud de los pacientes de atención primaria. Aten Primaria 2003; $32: 1-8$

3. Antolín A. Conocimientos acerca de su enfermedad y de los documentos de voluntades anticipadas en los pacientes con enfermedades crónicas evolutivas descompensadas que consultan en urgencias. Emergencias 2007; 19: 245-50.

4. Barrio I, Simón P, Judez J. De las voluntades anticipadas o instrucciones previas a la planificación anticipada de las decisiones. Nure Investigación 2004; 5: 1-9-anexo I (objetivos de la planificación anticipada) y anexo II (planificación anticipada de las decisiones)-

5. Couceiro A, Pandiella A. La EPOC: un paradigma para el uso de directivas previas y la planificación anticipada de decisiones. Arch Bronconeumol 2010; 46: 325-31.

Indicaciones

1. Para aprender cómo se realiza la planificación anticipada de decisiones, seleccionar un caso clínico y seguir el modelo que aparece en el artículo n.․ 5 del soporte bibliográfico.

2. El caso clínico elegido puede referirse a cualquier enfermedad crónica que permita la planificación anticipada de decisiones, a excepción de la enfermedad pulmonar obstructiva crónica. El alumno debe conocer su evolución, los episodios intercurrentes, las fases de la patología y las causas habituales que conducen a la muerte.

3. Además de estos textos, específicos de soporte para esta práctica, los alumnos deben utilizar el material bibliográfico correspondiente a la clase teórica de este tema.

damento ético, y si el clínico tiene alguna obligación consecutiva, como es dar una información comprensible, garantizar la voluntariedad del paciente y evaluar si este paciente comprende bien las consecuencias de la decisión que está tomando. 
Tabla VII. Bioética y final de la vida: práctica 3.

Objetivos

1. Identificar el marco clínico de los cuidados paliativos.

2. Aprender a utilizar el concepto de limitación del esfuerzo terapéutico en las enfermedades avanzadas y en las situaciones terminales.

3. Identificar los síntomas prevalentes en las enfermedades avanzadas y terminales, y conocer la existencia de protocolos clínicos que definen su abordaje paliativo.

4, Aprender a valorar, de manera profesional, el sufrimiento de los pacientes con este tipo de enfermedades y a desglosar las variables en las que se puede intervenir para atenuarlo.

5. Aprender a identificar las necesidades psicosociales del enfermo y de su familia, y conocer la existencia de estrategias profesionales de intervención.

Material de apoyo

1. Guías clínicas. Sociedad Española de Cuidados Paliativos (SECPAL).

2. Ameneiros $\mathrm{E}$, et al. Limitación del esfuerzo terapéutico en la situación de enfermedad terminal: ¿nos puede ayudar la bioética a tomar decisiones? Med Pal 2006; 13: 174-8.

3. Navarro N, Fombuena M. Cuidados paliativos en el enfermo no oncológico. In Consejería de Sanidad, Generalitat Valenciana. Atención Paliativa. Guía práctica n.ํ67. p. 6-7.

4. Soler $\mathrm{M}$, et al. Necesidades psicosociales de los enfermos al final de la vida, y de sus familiares. Cuidados paliativos en el enfermo no oncológico. In Consejería de Sanidad, Generalitat Valenciana. Atención Paliativa. Guía práctica n.ำ 67. p. 12-3.

5. Clavé $E$, Casado A. Sufrimiento insoportable y voluntad de vivir: primeros resultados de un estudio en Guipuzkoa. Med Pal 2006; 13: $197-206$.

Si aplicamos todo ello al diseño de una actividad práctica con los alumnos sobre el problema del rechazo de tratamiento de un paciente, construiremos un diálogo muy distinto al habitual por diversas razones:

- Los alumnos no podrán dar razones sin conocer en profundidad el tema sobre el que se está dialogando, cuyos contenidos teóricos ya tienen que haber trabajado de antemano.

- A diferencia de lo que suele ocurrir, aprenden que no vale 'cualquier opinión' y que sólo deben tenerse en cuenta opiniones argumentadas de personas que conocen aquello sobre lo que hablan.

- Identifican aquellos valores que la sociedad actual entiende que deben respetarse en la relación clínica, entre ellos la vida y la libertad, lo que permite superar la 'neutralidad axiológica' del 'todo vale'.

- Dados todos estos supuestos previos, aún queda ponderar, sopesar en diálogo con los otros, qué cursos o vías de actuación parecen los más adecuadas para responder al caso del paciente que rechaza un tratamiento. Es decir, aprender a ponderar con prudencia el caso concreto para saber qué valor priorizar y por qué hacerlo [29].

Es así como la educación deliberativa supera el pragmatismo tan extendido en el campo de la ética y se aleja de la confusión con la tolerancia [30]. La tolerancia es una forma de expresar el respeto a los demás aceptando sus diferencias; es la virtud que mejor caracteriza a las sociedades democráticas. Tolerancia es admitir en el diálogo todas las perspectivas y opiniones, pero de ahí no se puede deducir que cualquier opinión tenga el mismo valor para los interlocutores, ni tampoco para la sociedad plural en la que vivimos, que parte del respeto irrenunciable a los derechos humanos de primera y segunda generación.

La enseñanza de la ética que asume la tolerancia como virtud fundamental tiene que capacitar, al mismo tiempo, para identificar y señalar opiniones que no cumplen con los requisitos de la propia tolerancia o con los valores que la sustentan.

\section{Conclusión}

La enseñanza práctica de la bioética no se circunscribe exclusivamente a identificar problemas éticos, ubicarlos bajo el paradigma de los cuatro principios y discutir sobre ellos. Al igual que en la actividad clínica, se requieren ciertas habilidades específicas sin las cuales no es posible resolver con fundamento los problemas que plantea el marco cívico actual de la relación clínica. Adquirir estas destrezas y aprender a deliberar es la manera de capacitar a los alumnos de pregrado en este ámbito del conocimiento.

\section{Bibliografía}

1. Tysinger J, Klonish L, Sadler J, Wagner J. Teaching ethics using small-group, problem based learning. J Med Ethics 1997; 23: 315-8.

2. Couceiro A. Cinco mitos sobre la enseñanza de la bioética en las facultades de medicina. Rev Educ Cienc Salud 2009; 6: 68-74.

3. Couceiro-Vidal A. Enseñanza de la bioética y planes de estudios basados en competencias. Educ Med 2008; 11: 69-76.

4. Casado A. Bioética para legos. Una introducción a la ética asistencial. Madrid: CSIC/Plaza y Valdés; 2008.

5. Marzábal I. Deliberaciones poéticas. Cine y ética narrativa. Bilbao: Universidad del País Vasco; 2004.

6. Flexner A. Medical education in the United Status and Canada. New York: The Carnegie Foundation for the Advancement of Teaching; 1910.

7. González J, Wagenaar R. 'Tunning educational structures in Europe'. Informe final. Deusto: Universidad de Deusto; 2003. 
8. Couceiro A, Muñoz M. La enseñanza de la bioética en medicina. Una propuesta de desarrollo curricular. Rev Educ Cienc Salud 2007; 4: 92-9.

9. Couceiro A. Introducción a la bioética. In Rodés-Teixidor J, Guardia-Massó J, eds. Medicina interna. 2 ed. Barcelona: Masson; 2004. p. 16-21.

10. Gracia D. Planteamiento general de la bioética. In Gracia D, ed. Fundamentación y enseñanza de la bioética. Bogotá: El Búho; 1998. p. 11-28.

11. Simón P. Diez mitos en torno al consentimiento informado. An Sist Sanit Navar 2006; 29 (Supl 3): 29-40.

12. Comité Consultivo de Bioética de Cataluña. Recomendaciones ante el rechazo de los enfermos al tratamiento. Barcelona: Departament de la Salut, Generalitat de Catalunya; 2010.

13. Ley $41 / 2002$, de 15 de noviembre, básica reguladora de la autonomía del paciente y de derechos y obligaciones en materia de información y documentación clínica. Boletín Oficial del Estado 2002; 274: 40126-32.

14. Hernando P. Los derechos de los pacientes: una cuestión de calidad. Rev Calid Asist 2005; 20: 353-6.

15. Simón P, Barrio I, Alarcos F, Barbero J, Couceiro A Hernando P. Ética y muerte digna: propuesta de consenso sobre un uso correcto de las palabras. Rev Calid Asist 2008; 23: 271-85.

16. Couceiro A. ¿Es ético limitar el esfuerzo terapéutico? An Esp Pediatr 2002; 57: 5005-7.

17. Fernández R, Baigorri F, Artigas A. Limitación del esfuerzo terapéutico en Cuidados Intensivos. ¿Ha cambiado en el siglo Xxi? Med Intensiva 2005; 29: 338-41.

18. Iribarren S, Latorre K, Muñoz-Martínez T, Poveda Y, Dudagoitia JL, Martínez S, et al. Limitación del esfuerzo terapéutico tras el ingreso en una Unidad de Medicina
Intensiva. Análisis de factores asociados. Med Intensiva 2007; 31: 68-72.

19. Ley $2 / 2010$, de 8 de abril, de Derechos y garantías de la dignidad de la persona en el proceso de la muerte. Boletín Oficial de la Junta de Andalucía 2010; 88: 8-16.

20. Barrio I, Simón P, Judez J. De las voluntades anticipadas o instrucciones previas a la planificación anticipada de las decisiones. Nure Investigación 2004; 5: 1-9.

21. Couceiro A, Pandiella A. La EPOC: un paradigma para el uso de directivas previas y la planificación anticipada de decisiones. Arch Bronconeumol 2010; 46: 325-31.

22. Couceiro A. Las directivas anticipadas en España: contenido, límites y aplicaciones clínicas. Rev Calid Asist 2007; 22: 213-22.

23. The Hastings Center. Los fines de la medicina. El establecimiento de nuevas prioridades. Barcelona: Cuadernos de la Fundación Víctor Grífols; 2004.

24. Cherny N, Radbruch L; The Board of EAPC. European Association for Palliative Care (EAPC) recommended framework for the use of sedation in palliative care. Palliative Med 2009; 23: 581-93.

25. Comité Ético de la Sociedad Española de Cuidados Paliativos. Aspectos éticos de la sedación en cuidados paliativos. Med Pal 2002; 9: 41-6.

26. Charlesworth M. La bioética en una sociedad liberal. Cambridge: Cambridge University Press; 1993.

27. López de la Vieja MT. Bioética y ciudadanía. Madrid: Biblioteca Nueva; 2008.

28. Gracia D. La deliberación moral: el método de la ética clínica. Med Clin (Barc) 2001; 117: 18-23.

29. Couceiro A. La ética del diálogo en el mundo sanitario. JANO 2005; 1586: 72-4.

30. Gutmann A. La educación democrática. Una teoría política de la educación. Barcelona: Paidós; 2001 